UDK 821.131.1-96.09Bruno G. UDK 821.131.1-1.09Petrarca F.

\title{
TRA POLEMICA E RISEMANTIZZAZIONE: LA POSIZIONE DI G. BRUNO RISPETTO A PETRARCA, PETRARCHISTI, ANTIPETRARCHISTI
}

\author{
Patrizia Farinelli
}

\begin{abstract}
:
The gnoseological reflection of Giordano Bruno in De gli eroici furori (1585) is widely shaped by the use of expressive structures and images of the poetry which leads back to Petrarch and to the current referring to him. According to this hypothesis, the elements which explain this choice chiefly consist in structural analogies between the phenomenology of love as outlined in Petrarchan poetry and Bruno's conception of the relationship that connects a knowing subject and a known object (the infinite One). Even though Bruno in the end - against his intentions - highlighted exactly a certain philosophical value in Petrarch's lyrical poetry, he was not willing to recognize himself in any bond with that tradition. His radical rejection of Petrarch and those imitating and parodying him - on which prefatory pages are centered - particularly shows a necessity to differentiate between the actual object of inquiry and the most widespread profane lyrical poetry of his century (as well as the tout court lyrical poetry). Even leaving aside author's polemical position in order to rather comprehend what is evident from the text itself, the possibility to consider Bruno as much Petrarchist as Antipetrarchist nevertheless continues to elude. The reason for this is that adaptation and philosophical resemantization of that lyrical code are in Bruno's case set in a singular way compared to the then widespread relation between imitatio, inventio, emulatio; more generally, they are set outside of literary communication: that code primarily enters his work in cognitive function.
\end{abstract}

Key words: Giordano Bruno, Petrarchists, resemantization

\section{IN DIFESA DEL VALORE COGNITIVO DELLE IMMAGINI}

La scelta di affrontare una riflessione filosofica attraverso un discorso per immagini e dunque in una forma più letteraria che accademica è molto frequente nell'opus bruniano. Anche il suo scritto di argomento gnoseologico De gli eroici furori ${ }^{1}$ stilato come raccolta autocommentata di poesie (in prevalenza di sonetti), testimonia una simile prassi. Al discorso lirico e narrativo dei componimenti in versi, gli annessi commenti dialogati affiancano uno logico-argomentativo ben permeato a sua volta di

\footnotetext{
${ }^{1}$ Riprendo qui, puntalizzandole, delle tesi esposte in un precedente e più ampio lavoro (Farinelli 2000).

Nel seguito si citerà questopera dalla seconda ristampa della 3. edizione Sansoni dei Dialoghi italiani (Bruno 1985) e la si citerà sotto la sigla D.I.
} 
figuralità. Il bagaglio delle immagini è attinto alle più svariate fonti, sia sacre, che profane, ma centrale resta la semiotica improntante la lirica amorosa di Petrarca e dei petrarchisti. ${ }^{2}$

Composta e pubblicata nel periodo del soggiorno inglese del filosofo alla corte elisabettiana, ${ }^{3}$ l'opera tratta le modalità di ricerca e comprensione dell'Uno infinito o Causa prima che, come più alto oggetto di conoscenza e senza connotazioni sacre, appare anche sotto il termine di "divino". L'autore v'illustra più specificamente lo stato, non privo di dubbi e cedimenti, in cui verrebbe a trovarsi chi s'inoltra in una simile ricerca tra l'anelito a cogliere l'infinito e la consapevolezza dei limiti umani d'ostacolo alla sua comprensione. In preda a sentimenti contrastanti, ma interiormente vincolato al suo fine, il soggetto di tale impresa, che il nolano recuperando e attualizzando la teoria platonica dei furori vuole come "eroico furioso" ${ }_{4}^{4}$ può solo tornare sui risultati parziali raggiunti nella ricerca e mantenerli aperti attraverso un processo destinato a non trovare fine, proprio nel senso della conoscenza che attraverserà la modernità. ${ }^{5}$

Rispetto alla struttura testuale, uno dei fenomeni più vistosi del lavoro è costituito dalla presenza di successive variazioni nell'organizzazione delle rime. Mentre la sezione iniziale, rappresentata dai primi quattro dialoghi, si presenta come un canzoniere commentato, quelle che seguono, senza che sia mai abbandonata la strategia del commento, assumono rispettivamente la forma di un libro d'imprese, di un contrasto allegorico, di una raccolta di sonetti a corona e infine di un racconto mitologico: queste sezioni svolgono la funzione di ripetere, specificare e amplificare quanto esposto nei dialoghi iniziali. La presenza dominante della variazione, visibile del resto in più aspetti formali e non solo sul piano architettonico, non stupisce. ${ }^{6}$ Concorda anzi sia col valore assegnato dal filosofo alla facoltà dell'inventio, sia con la sua posizione poetologica fondamentalmente antinormativa. Non è tuttavia sulla complessa struttura testuale, già considerata in altra occasione, che ci si vuole soffermare qui. ${ }^{7}$ L'intenzione è invece di focalizzare come si profili il rapporto dell'autore verso Petrarca e la corrente che vi fa capo. Si considereranno dapprima, dunque, quali ragioni potessero indurre Bruno a scrivere dei trattati filosofici adottando in ampia misura un linguaggio figurale per indagare poi i motivi che lo spinsero a puntare (per quella specifica tematica) proprio sul codice espressivo e concettuale della poesia amorosa e in particolare su quella di tradizione petrarchesca.

Non escludendo che ci fossero anche delle ragioni socioculturali a suggerire al nolano d'illustrare il processo di conoscenza dell'infinito nelle forme e immagini proprie

\footnotetext{
${ }^{2}$ Tra le opere profane del suo secolo, l'autore tiene presente la tragicommedia Cecaria (1525) di Marc'Antonio Epicuro.

${ }^{3}$ Lopera fu pubblicata a Londra nel 1585, ma per motivi verosimilmente cautelari sotto falsa indicazione di luogo (Parigi). È dedicata al poeta Philip Sidney. Ė divisa in due parti di cinque dialoghi ciascuno. Su 72 componimenti in versi di Bruno, in essa presenti, 67 sono sonetti. Compresi in forma di citazione sono anche 4 sonetti di Tansillo.

${ }^{4}$ Bruno si riferisce a un furore non innato, bensì raggiunto attivamente attraverso un processo razionale e una volontà di conoscenza. Cfr. D. I.: 987.

${ }^{5}$ Sul problema della conoscenza in Bruno cfr. Spruit 1988.

${ }^{6} \mathrm{Tra}$ le variazioni di superficie anche quella metrica. Quasi a siglare con una cifra individuale i propri versi, Bruno introduce nelle terzine due settenari. Per le caratteristiche relative a metro e rima nelle poesie di questopera cfr. Sabbatino 1993.

${ }^{7}$ Farinelli 2005.
} 
di un codice letterario assai diffuso nelle cerchie laiche in cui operava in quegli anni, ${ }^{8}$ è plausibile che vi svolgessero un ruolo determinante delle motivazioni intrinseche al suo sistema di pensiero. Occorre ricordare al proposito l'atteggiamento di rifiuto mostrato da Bruno nei confronti dell'aristotelismo anche in ambito gnoseologico ${ }^{9}$ e la difesa, invece, della tesi di platonica memoria, rivisitata anche dal Cusano, circa l'umbratilità del conoscere che veniva ribadita con energia in De gli eroici furori $(1585)^{10}$ dopo essere stata sostenuta in De umbris idearum (1582) e nel Sigillus sigillorum (1583). Nella gnoseologia bruniana il più alto oggetto di conoscenza, l'Uno o Causa prima, resta un principio trascendente ('S' io chiamo, non risponde;/ E quant'io cerco piú, piú mi s'asconde ${ }^{11}$ ) e si rende manifesto solo in forma mediata come quell'altro di sé che è l'infinito nella Natura, cioè nel processo della vicissitudine, non nella sua assolutezza. A valorizzare la funzione non solo euristica, ma anche cognitiva e mnemonica del discorso figurale si aggiungevano delle considerazioni sulle facoltà cognitive umane. Bruno, condividendo posizioni diffuse in ambito tardo rinascimentale come rilevano, fra altri, gli studi di Frances Yates e Lina Bolzoni, osservava che il nostro intelletto non è in grado di cogliere le cose nella loro semplicità e unità, ma solo nella combinazione. Nell'epistola dedicatoria del De imaginum, signorum et idearum compositione, un testo del 1591 che riprende nei concetti e nel metodo alcuni suoi precedenti scritti sull'arte mnemonica, specificava inoltre che l'intelletto umano non può comprendere se non in "specie, simulacro, imagine, figura, signo". ${ }^{12}$

\section{COL CODICE DELLA POESIA D'AMORE}

Se tali considerazioni si lasciano estendere anche ad altri scritti di Bruno, il fatto che questi nel caso specifico assuma proprio un codice espressivo e concettuale amoroso per trattare di gnoseologia si spiega - così ipotizziamo - col ruolo stesso che l'amore possiede nel suo sistema di pensiero quale forza basilare del processo conoscitivo. Entro tale orizzonte è esclusa la possibilità di cogliere l'infinito con le sole potenzialità della facoltà intellettiva se quest'ultima non è sostenuta da quella appetitiva. Spetta allora all'amore quale "furore eroico", quale fervore che si svilupperebbe durante il processo, mantenerne aperta la ricerca attivando sempre di nuovo l'uso della facoltà razionale. ${ }^{13}$ Bruno raccoglie certamente anche alcune esperienze rinascimentali di trattazioni filosofiche affrontate nei termini di un discorso d'amore (si pensi all'opera di Ficino De amore o ai Dialoghi d'amore di Leone Ebreo, usciti nel 1535 postumi), tende tuttavia a evitare l'uso d'immagini di discesa e ascesa diffuse nel neoplatonismo

\footnotetext{
${ }^{8}$ Sul peso del colto ambiente londinese nelle scelte di Bruno in fatto di lingua, genere e registro discorsivo richiamavano l'attenzione Aquilecchia (1953: 165-189) e Blum (1981: 685-692).

${ }^{9} \mathrm{~A}$ simili conclusioni conducono, in particolare, considerazioni presenti nel penultimo dialogo della raccolta. "Chi ha buon senso e non vede del profitto che fe' Aristotele [...] volendo con il suo raziocinio logicale ponere definizioni, nozioni, certe quinte entitadi ed altre parti ed aborsi de fantastica cogitazione per principii e sustanza di cose [...]?'D. I.: 1114-1115.

${ }^{10}$ Cfr. ivi: $1158-1159$.

${ }^{11}$ Ivi., p. 973.

${ }^{12}$ Bruno 1889: 91.

${ }^{13}$ All'interazione di facoltà intellettiva e appetitiva (occhi e cuore) è dedicato il quarto dialogo della prima parte.
} 
fiorentino, rappresentanti il rapporto tra finito e infinito in termini di successiva derivazione. In Ficino le fasi di approfondimento della conoscenza corrispondevano ad altrettanti gradi dell'essere, ma questi gradi spariscono nel sistema metafisico bruniano in cui tutto è invece pensato sussistere della stessa sostanza. Ogni possibile analogia fra stadi del conoscere e ordine gerarchico dell'essere, un ordine basato sulla maggiore o minore vicinanza a Dio, unità indifferenziata, viene così necessariamente a cadere spianando la strada a un nuovo paradigma di pensiero. ${ }^{14}$ Bruno mantiene il concetto di gradualità del conoscere solo nel senso che tale processo prevede svariati tentativi e un rapporto di assiduità, ma lo svincola radicalmente da una concezione gerarchica dell'essere. Quanto ribadisce, e in forma svariate, è che non si conosce per stadi ma istantaneamente. La conoscenza dell'infinito, seppur abbia luogo nella sua ottica solo quando si siano create le condizioni per il suo possibile accadere, è pretesa infatti come qualcosa di folgorante ${ }^{15}$ d'indipendente dalla sola volizione ${ }^{16}$ e tale da innescare una trasformazione nel soggetto coinvoltovi. Non da ultimo è concepita come un'esperienza che segue una misura individuale. ${ }^{17}$ Risulta comprensibile, su simili presupposti, che la dinamica amorosa sottesa alla lirica di Petrarca, col relativo bagaglio di concetti e d'immagini, potessero entrare bene in gioco nel sistema di pensiero bruniano per configurare il processo conoscitivo. Tale dinamica riceve però un adattamento in alcuni suoi aspetti ${ }^{18}$, in piccola parte viene anche contaminata con altre tradizioni ${ }^{19}$, ma prima di tutto subisce una radicale risemantizzazione, a qual fine è basilare il commento autorale. Nell'operazione selettiva sono tralasciati i motivi del rammarico e del pentimento (centrali invece nella poesia di Petrarca) poiché incongruenti con il senso della ricerca del divino; in ragione della portata sovra-individuale del discorso appaiono inoltre rari o spostati nel peritesto (ad esempio nel sonetto giustificativo) dei richiami storico-temporali. Analogamente è assente nei sonetti, e sporadico nelle parti in prosa, il motivo del ricordo. Aspetto tutt'altro trascurabile è poi il fatto che tanto i versi quanto i relativi commenti restino privi di riferimenti metapoetici.

Ciò che è decisivo della lirica di Petrarca, per un confronto con le rime bruniane sul furore eroico, non è solo il fatto che essa dia voce a un amore per un oggetto irraggiungibile, vissuto - così si pretende - con sentimenti antitetici da parte dell' amante e come un rapporto vincolante, quasi ossessivo. È ancor più decisivo il fatto che tale lirica canti un oggetto del desiderio che resta inaccessibile e fondamentalmente assente o meglio si renda presente all'io lirico solo in forma mediata: nei $R V F$ l'amata trova infatti una forma di presenza in ciò che resta legato per vicinanza fonetica al suo nome, al suo ricordo, al sogno che la evoca o ancora ritorna alla mente dell'io lirico

\footnotetext{
${ }^{14}$ Cfr. Blumenberg 19892.

${ }^{15}$ D. I.: 1157.

${ }^{16}$ Il processo prevede un apprendere e un essere preso; in temini figurali si legge: "Cacciatrice di me, la mia Diana". Ivi: 1112.

${ }^{17}$ Sulla misura individuale di ogni singola esperienza di conoscenza cfr. D. I.: 1029.

${ }^{18}$ Anche alcune scelte lessicali trovano ragione in funzione di un discorso d'amore non profano. L'oggetto cui tende l'eroico furioso è indicato da espressioni indeterminate, tali da evitare rimandi a un essere terreno (fra queste ricorrono: "beltà sola", "alta bellezza", "mio bene", "mia diva, "mia dea") o da sottolineare la natura figurale della sola forma comprensibile del divino ("immagine", "effigie").

${ }^{19}$ Sono presenti richiami biblici, riferimenti al concetto di unione mistica, al motivo cabalistico della morte del bacio nonché a miti classici (riprese dai salmi e dalla mitologia classica fanno parte del resto di una prassi anche petrarchesca).
} 
in modo associativo attraverso oggetti, luoghi, miti che la sostituiscono. Petrarca canta dunque la donna fondamentalmente in figura, attraverso i suoi simulacri. Le tematiche dell'assenza dell'oggetto desiderato, dell'anelito a qualcosa che resta lontano per ritornare alla mente dell'io lirico in forma mediata fino ad entrare in un rapporto di sostituzione con la parola che ne parla, sono centrali nel sistema lirico petrarchesco. Su quest'aspetto ha scritto delle pagine assai acute Adelia Noferi che insisteva appunto sul circolo che viene a crearsi nei $R V F$ tra parola del desiderio e desiderio della parola. ${ }^{20}$

\section{CONTRO LA POESIA PROFANA}

Tanto nel processo conoscitivo concepito da Bruno, quanto nel rapporto d'amore cantato da Petrarca, l'inaccessibilità dell'oggetto ne rafforza il desiderio comportando nella sua ripetuta ricerca anche il rischio di una perdita di sé da parte del soggetto coinvoltovi. Si tratta di due processi appartenenti a contesti fenomenologici diversi, che configurano però in modo analogo la ricerca dell' Altro (ivi compreso, come appena osservato, il modo traslato dell' Altro di evidenziarsi). Nella riflessione dell'io petrarchesco ciò che diviene presenza è essenzialmente la parola; mentre nella ricerca dell'eroico furioso a poter essere intellettualmente raggiunto è 'l'altro di sé' dell'Uno infinito ovvero l'infinito nel tempo che il soggetto conoscente scopre nella Natura e in se stesso quale essere appartenente a tale dimensione. Riprendendo l'esempio proposto dal più noto sonetto dell'opera, l'Atteone cacciatore si riconosce preda. ${ }^{21}$

Ciò che si viene attuando nell'opera lirica di Petrarca - lo schiudersi della parola poetica nella tensione verso qualcosa d'irraggiungibile - non è cosi lontano, nella dinamica processuale, dai risultati cui sfocia la ricerca dell'infinito nel sistema filosofico bruniano. In entrambi i casi ne va di un oggetto che si rende presente come altro da sé - un processo epifanico non conseguito come atto volontaristico del soggetto, sebbene imprescindibile dal suo cercare. Nell' attingere alle strutture di quella poesia d'amore, una poesia a cui in ogni caso, come sottolineava Contini, è centrale la dimensione dello scorrere del tempo, il nolano finiva contro le sue intenzioni per metterne in luce una valenza metafisica, ma dai suoi presupposti, dal suo diverso rapporto verso la parola poetica era escluso che riconoscesse tale componente. Anzi, nell'Argomento proemiale tesse una feroce invettiva contro il poeta di Laura, accusandolo di essersi abbassato a inseguire un amore terreno, così come attacca, con le stesse motivazioni, petrarchisti e antipetrarchisti al punto da azzerare allo stesso tempo anche la diversità di singole poetiche, singoli intenti e risultati. ${ }^{22}$ A muovere quella polemica, dove anche la lirica di Petrarca è ridotta a gioco d'ingegno attuato "con esplicar gli affetti d'un ostinato amor volgare, animale e bestiale [...]", è la necessità di difendere la peculiarità del suo discorso rispetto a quello della poesia profana in generale cui rimprovera la mancanza di un oggetto degno di speculazione. Bruno giudica dunque moralmente il valore della poesia in base all'altezza del suo oggetto. Riserva infatti maggior rispetto alla

\footnotetext{
${ }^{20}$ Noferi 1979: 69-209.

${ }^{21}$ Per una lettura filosofica del mito di Atteone nei Furori cfr. Beierwaltes 1979. Per un confronto fra l'elaborazione di quel mito in G. Bruno in G. B. Marino cfr. Farinelli 2012.

${ }^{22}$ Esiste ora una stampa del solo L'Argomento cui è annesso un dettagliato commento (Canone 2012).
} 
poesia spirituale, cui fa brevemente riferimento menzionando il Cantico dei cantici, ma non manca di differenziare la propria anche da quella, sottolineando il suo intento specificamente filosofico.

\section{A DISTANZA DA PETRARCA, PETRARCHISTI E ANTIPETRARCHISTI}

Bruno era lontano da esigenze di carattere estetico nello stendere le sue rime sul furore. Nei suoi sonetti, di cui riassumiamo qui le caratteristiche principali, la parola non è promossa a creare né emozione né stupore, né interrogazione, ma semmai a esemplificare. Raramente viene a crearsi una densità di rapporti fra gli elementi che le compongono, tale da rendere possibili sensi plurimi. La stessa presenza del commento autorale tende del resto a congelarne altre possibili interpretazioni. Al confronto le liriche del conterraneo Luigi Tansillo, cui Bruno fa talvolta riferimento diretto nel suo discorso, presentano una ben maggiore carica speculativa. ${ }^{23}$

I sonetti del nolano mostrano per lo più strutture logico-sintattiche ripetitive, $\mathrm{i}$ cosiddetti versi rapportati $;{ }^{24}$ in qualche altro caso seguono invece quell'ordine oppositivo che trova un modello nel sonetto petrarchesco "Pace non trovo": ne sono degli esempi l'ottavo sonetto del II dialogo come pure le terzine di un sonetto del III dialogo, uno dei meglio riusciti della raccolta sia per l'agilità con cui si sviluppa il discorso sia per la stretta corrispondenza tra registro semantico (leggero, pesante; alto, basso) e registro sintattico:

Altr'amo, odio me stesso;

Ma s'io m'impiumo, altri si cangia in sasso;

Possi'altr'al cielo, s'io mi ripogno al basso;

Sempr'altri fugge, s'io seguir non cesso;

E s'io chiamo, non risponde;

E quant'io cerco più, più mi s'asconde..$^{25}$

La figura retorica dominante, come attendibile, è l' antitesi, che appare spesso nella forma di ossimoro e in formule diffuse ("dolce languire", "sordi affanni", "amaro diletto e dolce pena"; "odioso bene", "dolce danno", "dolce nemico"). Quanto alle immagini adottate, Bruno ricorre spesso a quella topica del fuoco ("fiamme", "face", "vampo", "ardore") per parlare vuoi dell'amore e dell'oggetto stesso, vuoi della condizione del soggetto che si cimenta in quella ricerca; rende inoltre il legame che l'amante prova verso l'amata (rispettivamente il soggetto conoscente e l'Uno infinito) con espressioni radicate nella poesia petrarchesca come "lacci", "catene", "giogo". Quanto all'oggetto d'amore, lo indica non di rado attraverso l'immagine degli occhi (anche nelle varianti "luci" e "lumi"). Altre immagini ricorrenti sono quelle della guerra e della cecità, assunte in funzione di rendere rispettivamente le modalità della ricerca e lo stato di strutturale inadeguatezza di

\footnotetext{
${ }^{23}$ Tansillo si profila come poeta raffinato con uno stile personale. Cfr. Afribo 1994. Tansillo entra in Degli eroici furori anche come figura di dialogante. Sulla presenza dell'opera di Tansillo in Bruno cfr. Rowland 2003.

${ }^{24}$ Cfr. D. I.: 964 (son 4. nel dial. I/1, vv. 1-6). Ha richiamato lattenzione su questa peculiarità dei sonetti bruniani Mönch 1955: 76-78.

${ }^{25}$ D. I.: 973 (son. 1 nel dial. I/2, vv. 9-14).
} 
chi la compie. ${ }^{26}$ Anche le immagini del viaggio e del volo rappresentano la ricerca dell'alto oggetto mentre quelle legate al cibo, nella fattispecie l'impossibilità di saziarsi, definiscono l'inesauribilità della sua ricerca. La ruota indica il movimento che regola la natura. Fra le poche immagini di luoghi, appare quella del bosco impraticabile (gli "erti sentieri") che non rappresenta tuttavia un locus terribilis, bensì lo stato di positivo isolamento dell'eroico furioso quale presupposto alla ricerca. Raramente vi emergono effetti fonetici di qualche intensità ad eccezione forse di certe paronomasie ("m'appaga"/"impiaga"). Più interessanti e più personali risultano invece le scelte lessicali. Al proposito va rilevato l'uso di termini del linguaggio parlato e di vocaboli esprimenti concretezza per descrivere uno stato interiore. Si pensi a espressioni come "liquefaccio intero" o "sto a continue botte" o ancora "chi la mente m'ingombra è un sol viso". ${ }^{27}$

L'esigenza di confrontarsi specificamente sul piano letterario con Petrarca è in Bruno assente. Anche preoccupazioni stilistiche cadevano evidentemente in secondo piano nella stesura di quelle rime rispetto all'intento di performare con chiarezza la propria concezione della ricerca del divino, una concezione che partiva dal superamento della metafisica neoplatonica, ancora fissata al concetto di derivazione dell'essere per gradi progressivi di allontanamento dalla perfezione divina, e poneva le basi di una metafisica in cui non si distingue più tra materia finita e materia infinita, ma solo tra diverse forme dell'essere.

Per dar voce al suo pensiero il filosofo attivò delle strategie che continuano a stupefarci e su di esse merita indagare restando quanto possibile liberi da un tentativo di classificare l'operazione da lui compiuta. Una considerazione di carattere storico-letterario porta però inevitabilmente a chiedersi se il Bruno dei Furori, assumendo elementi della poesia che faceva riferimento a Petrarca per adattarli e risemantizzarli alle proprie esigenze filosofiche, entri a far parte del sistema letterario del petrarchismo, caratterizzato dalle dinamiche (non necessariamente sempre unite) di imitatio, inventio et emulatio, o vi resti estraneo. La questione non è lineare perché anche in lui ci fu e ci fu rinnovamento, ma un rinnovamento consistente in trasposizione su un altro piano semantico, mentre l'emulazione e altrettanto la parodia furono del tutto assenti. Considerare Bruno come un rappresentante di quel sistema è problematico nonostante il petrarchismo fosse un fenomeno plurimo e il rifarsi a Petrarca dopo la metà del Cinquecento significasse accogliere in maniera sempre maggiore la variazione. Quondam ricordava le trasformazioni visibili nella produzione lirica napoletana a quell'altezza temporale, in cui era visibile un allontanamento dal modello all'interno del modello stesso attraverso un esautoramento delle possibilità che questo offriva senza rappresentarne ancora un definitivo superamento. ${ }^{28} \mathrm{De}$ gli eroici furori resta però un lavoro difficilmente integrabile in quel tipo d'interventi

${ }^{26}$ Il concetto di cecità è nel testo plurisignificante; nell'ultimo dialogo, con riferimenti alla Cecaria, indica anche l'inizio della vita intellettiva e trova allora corrispondenze con quel concetto di morte come raggiungimento di un nuovo stato conoscitivo, illustrato sia dall'episodio mitologico della fine di Atteone, sia dall'immagine di tradizione cabalistica della morte del bacio.

${ }^{27}$ Rispettivamente D. I.: 1080 (son. 3 nel dial. II/1, v. 14); 1064 (son. 13 nel dial. I/5, v. 8); 963 (son. 3 nel dial. I/1, v. 10).

${ }^{28} \mathrm{Cfr}$. Quondam (1975: 63-186) osserva che le opere composte in quei decenni si distinguono soprattutto per fenomeni di accumulo e di amplificazione a livello retorico e per la scelta di nuovi criteri organizzativi per le raccolte di poesie. 
poiché non si realizza affatto come un'attività e una riflessione di carattere letterario. Altrettanto poco convincente è considerarla opera di un antipetrarchista. Bruno fu sì antiaccademico e insofferente verso qualsiasi modello, però l'intervento effettuato non ha nulla di quello degli antipetrarchisti. Anche lui difendeva lo strappo dalla norma, il gesto poetologicamente trasgressivo come l'uso di registri espressivi diversi e non di rado bassi, la sovrapposizione di generi, l'accostamento di fonti disparate nello stesso contesto. Gli antipetrarchisti restavano tuttavia in un rapporto comunicativo, seppur polemico e/o ludico, con Petrarca e con i petrarchisti (che furono un loro obiettivo di attacco più di quanto lo fosse Petrarca stesso), mentre Bruno muoveva da ben altre intenzioni. Non fu tale, però, da proporre un nuovo paradigma di poesia.

Che il rapporto del filosofo verso Petrarca non fosse precisamente quello dei petrarchisti è anche la tesi sostenuta da Florian Mehltretter in un articolo in cui presenta l'operazione compiuta dal nolano come una trasformazione della struttura profonda della poesia di Petrarca che comporta ai suoi occhi, come fenomeno centrale, l'instaurarsi di un diverso rapporto fra soggetto-oggetto: mentre nei $R V F$ è solo il soggetto esposto a una condizione di scissione interiore, in De gli eroici furori tale condizione non è unicamente del soggetto ma anche dell' oggetto. ${ }^{29} \mathrm{Si}$ potrebbe osservare al proposito che non è del tutto corretto parlare in termini di scissione e tantomeno di contraddizione nel caso dell'oggetto verso cui tende il furioso. In gioco è un rapporto di unità nel doppio; Bruno insiste appunto a delineare l'infinito fuori dal tempo e l'infinito nel tempo due modalità del medesimo. Particolarmente istruttiva nell'argomentazione di Mehltretter è piuttosto l'osservazione secondo cui la questione di comprendere come Bruno si situi rispetto alla lirica petrarchesca andrebbe posta considerando quale definizione di petrarchismo, fra quelle proposte in ambito storico-letterario, spieghi meglio l'operazione effettuata in De gli eroici furori. Alle definizioni di petrarchismo rispettivamente come relazione contraddistinta dall'assunzione di singole forme del modello, come dialogo col caposcuola o ancora come un sistema comportante un'attualizzazione del modello, preferisce una quarta, secondo la quale il petrarchismo si caratterizzerebbe allo stesso tempo come un dialogo metapoetico con il caposcuola (una relazione di vicinanza) e come innovazione (una relazione di distanza) ${ }^{30}$ Da parte sua Bruno dunque attualizzerebbe da un lato la lirica di Petrarca, dall'altro non entrerebbe per nulla in dialogo con costui - da qui l'assenza di riferimenti metatestuali; in questo senso egli entrerebbe nello stesso gioco cui prendono parte anche i petrarchisti, ma per condurlo in altro modo. ${ }^{31}$

La singolare posizione tenuta da Bruno rispetto a Petrarca, petrarchisti e antipetrarchisti evidenzia dal punto di vista metodologico che il tentativo di contestualizzare opere innovatrici con categorie rigide d'inclusione o esclusione da un sistema di riferimento diventa una forzatura. Considerata in rapporto all'opus del filosofo, questa posizione permette di cogliere, più in generale, il suo libero modo di lavorare sulle fonti, un modo che sembra mettere in pratica una tesi formulata proprio in De gli eroici furori secondo cui ogni processo conoscitivo ha necessariamente la propria misura.

Università di Lubiana, Slovenia

\footnotetext{
${ }^{29}$ Mehltretter 2004: 168.

${ }^{30}$ Ivi: 174. Mehltretter rimanda al proposito alle tesi di A. Kablitz.

${ }^{31}$ Mehltretter 2004: 177.
} 


\section{BIBLIOGRAFIA}

\section{FONTI:}

Bruno, Giordano. Dialoghi italiani, con le note di Giovanni Gentile, cur. Giovanni Aquilecchia. Firenze: Sansoni $1985^{3}$.

De umbris idearum, ed. crit., cur. Rita Sturlese. Firenze: Olschki, 1991.

Opera latine conscripta, vol. II, 3., cur. F. Tocco, H. Vitelli. Firenze: Le Monnier, 1989.

Tansillo, Luigi. Canzoniere edito ed inedito. Secondo una copia dell'autografo e altri manoscritti e stampe, voll. I-II, introduzione e note di Erasmo Pèrcopo, cur. Tobia R. Toscano. Napoli: Liguori, 1996.

\section{STUDI:}

Afribo, Andrea. "La ripetizione e altro in Luigi Tansillo", Rivista di letteratura italiana, 12 (1994): 351-382.

Aquilecchia, Giovanni. Le opere italiane di Giordano Bruno. Critica testuale e oltre. Napoli Bibliopolis 1991.

Beierwaltes, Werner. Actaeon. Zu einem mythologischen Symbol Giordano Brunos, Zeitschrift für philosophische Forschung, 32 (1979): 345-354.

Blum, Paul Richard. Giordano Bruno am englischen Hof, in Europäische Hofkultur im 16. und im 17. Jahrhundert «Wolfenbüttler Arbeiten zur Barockforschung», cur. August Buck, vol. III. Hamburg: Hanswedell 1981: 685-692.

Blumenberg, Hans. Kein kopernikanisches Martyrium: Giordano Bruno, in Id., Die Genesis der kopernikanische Welt. Teil III. Frankfurt a. M.: Suhrkamp 1989²: 416-502.

Canone, Eugenio. L'Argomento degli Eroici furori di Bruno. Pisa-Roma: Serra 2012. Supplementi, XXXI (Materiali, 6) di Bruniana \& campanelliana.

Farinelli, Patrizia. Il furioso nel labirinto. Studio su De gli eroici furori di Giordano Bruno. Bari: Adriatica 2000.

"Quando Proteo è l'opera stessa. Trasformazioni interne del testo in De gli eroici furori e ne La sampogna", in Metamorphosen, Akten des 6. Jahrestages des D.I.V. (Dresden 8-10 November 2001), cur. Peter Kuon, Barbara Marx. Frankfurt a. Main: Lang 2005: 113-131. "Izpod peresa dveh antiklasicistov: mit o Akteonu pri G. Brunu in G. B. Marinu". Keria: studia latina et graeca, 14, 2 (2012): 33-49.

Mehltretter, Florian. Giordano Bruno und der Petrarchismus. Bemerkungen zum ersten Teil der Eroici furori, Romanistisches Jahrbuch, 54 (2003)/Berlin-New York: De Gruyter 2004: 146-179.

Mönch, Walter. Das Sonett. Gestalt und Geschichte. Heidelberg: Kerle 1955.

Noferi, Adelia: Il gioco delle tracce. Studi su Dante, Petrarca, Bruno, il Neo-classicismo, Leopardi, l'Informale. Firenze: La Nuova Italia 1979.

Quondam, Amedeo. La parola nel labirinto. Società e scrittura nel Manierismo a Napoli. Bari: Laterza 1975.

Rowland, Ingrid. “Giordano Bruno e Luigi Tansillo", Bruniana \& campanelliana, IX, 2 (2003): 345-355

Sabbatino, Pasquale. Giordano Bruno e la «mutazione» del Rinascimento. Firenze: Olschki 1993.

Spruit, Leen. Il problema della conoscenza in Giordano Bruno. Napoli: Bibliopolis 1988. 